\title{
Performance Evaluation of Policy-Based Admission Control Algorithms for a Joint Radio Resource Management Environment
}

\author{
S. Luna Ramírez, M. Toril Genovés, M. Fernández \\ Navarro \\ Dpto. Ingeniería de Comunicaciones \\ E.T.S.I. Telecomunicación. Universidad de Málaga \\ Málaga, Spain \\ \{sluna, mtoril,mariano\}@ic.uma.es
}

\author{
Ronan Skehill, Sean McGrath \\ Wireless Access Group \\ University of Limerick \\ Limerick, Ireland \\ \{ronan.skehill, sean.mcgrath\}@ul.ie
}

\begin{abstract}
In this paper, several Admission Control (AC) algorithms are presented and evaluated in a Joint Radio Resource Management (J-RRM) environment where several Radio Access Technologies (RATs) coexist. The considered policy-based AC algorithms make use of load-balancing concepts. Performance assessment is based on simulations. During the analysis, the AC methods were compared against the non-joint AC scheme, which was considered as a benchmark. Results show that overall network behavior and the best $\mathrm{AC}$ algorithm prove dependent on coverage radius, terminal capabilities and network capacities. Nonetheless, results show that, in some cases, AC algorithms for JRRM schemes can reach $60-80 \%$ traffic gains when compared to the non-joint scheme.
\end{abstract}

\section{INTRODUCTION}

Fast growth and evolution in mobile communications and networks have been reflected in different areas. The penetration rate of mobile communication systems have now reached all time highs across Europe. However, cellular technology has not been the only Radio Access Technology (RAT) that has enjoyed success over the past decade. In parallel, wireless data networks, such as WLAN, have been gaining significant ground. As a consequence, the rollout of various RATs has not necessarily been an ordered and uniform one, as there has not been a focus on a single technology that is widely used and accepted. As a result, networks consisting of multiple Radio Access Technologies have arisen and these RATs are currently controlled independently.

In these networks that comprise several RATs, a common pool of resources can be created and be used for sharing purposes. This would allow users to obtain higher levels of Quality of Service (QoS) from the network and allow the network to make efficient decisions on admitting users to one of the RATs. This would be desirable not only to increase coverage and traffic capacity, but also to get a better adaptation between services and RATs. This strategy goes towards a more logical use of radio resources from different RATs resulting in a more economic and efficient solution, which is the main focus of Joint Radio Resource Management (J-RRM).
Admission Control (AC) is an initial part of J-RRM strategies that admits or rejects a connection depending on the network capacity. In J-RRM, AC considers the different RAT resources as a single radio set for the user connection. Current RATs, such as GSM, use higher-level entities to manage resources in the whole coverage area. In J-RRM, higher-level entities must know the network state for each technology in order to take a global (and possibly better) decision, which will allow the AC to send the user to the most appropriate RAT. Such a concept is referred to as Joint Session Admission Control [1].

Different contributions related to general J-RRM issues can be found in literature. Reference [1] makes a wide analysis of J-RRM concepts over a classical mobile network structure and proposes some new entities with their functionalities. $3 \mathrm{GPP}$ has defined several grades of integration between RATs in a heterogeneous scenario, including Wireless Local Area Network (WLAN) technology [2]. In [3], a clear distinction is made between possible J-RRM schemes, depending on that grade of integration between RATs. Regarding the algorithms to select the RAT to which every user is attached, two main types have been suggested. Policy-based controllers [4] use 'if-then' rules that take the decision based on crisp values of network performance indicators (e.g.: if 'active connections $>100$ ', then 'start handover'). In spite of its simplicity, these controllers suffer from several limitations that can be solved by J-RRM fuzzy logic controllers [5]. Additionally, [6] shows some analytical results using Markov chains for an early resource-sharing model; and [7] describes intersystem handover $(\mathrm{HO})$ between UMTS-FDD and TDD using similar schemes.

This paper presents different AC policy-based algorithms in a J-RRM scenario model over multiple RATs, using policy-based rules. A simulation model is presented, which captures the general behaviour of different joint management techniques. This model, together with software simulations, is used to test several new proposals for AC algorithms. The paper shows how AC policy based algorithms can be used in managing joint resources and highlights the advantages.

This work has been partially funded by the Spanish Ministry of Science and Technology, project TIC2003-07827. 
Important conclusions about network parameter configurations and strategy selection depending on scenario characteristics are also obtained. The rest of the paper is organized as follows. In section II, a general scheme is presented together with the proposed AC algorithms. This scheme is later used to compare the behaviour of J-RRM techniques against the non-joint (so called, 'reference') scheme. Section III describes the simulation setup implementing previous J-RRM schemes and AC algorithms. Key parameters and several scenarios are defined and tested with penetration indexes and terminal capabilities variations. Software implementation allows a wide flexibility and testing possibilities, both for algorithm definition and parameter settings. Section IV discusses the results and the main conclusions are finally presented.

\section{GENERAL SCHEME AND AC ALGORITHMS}

Fig. 1 represents the J-RRM scenario considered in this work. The network topology has a policy-based J-RRM entity which implements the AC tasks in the heterogeneous network. New connections ask for a RAT to manage the requested service. The J-RRM entity follows the policy previously defined for it and takes the final decision based on incoming connection characteristics and RATs statistics (mainly load). Whatever the final decision is, the connection is then assigned to that RAT.

Incoming connection has a $\mathrm{RAT}_{\text {home }}$ configuration which means the preferred RAT to be assigned to that User Equipment (UE). This concept will be used in the definition of the proposed AC algorithm.

Four different AC algorithms are defined in this paper $\left(\mathrm{AC}_{\text {type }}=1 . .4\right)$. The two first options deal with the $\mathrm{RAT}_{\text {home }}$ concept and the two remaining ones do not. Figures 2 and 3 represent the logic behind these two sets of strategies. $\mathrm{AC}_{\text {type }}=1$ only attempts to connect with its radio technology. As observed in Fig. 2, the connection will be blocked if UE is out of coverage or there are no radio resources available in $\mathrm{RAT}_{\text {home. }}$ When an attempt to $\mathrm{RAT}_{\text {home }}$ has failed, this scheme does not redirect connections to another RAT but counts them as blocked.

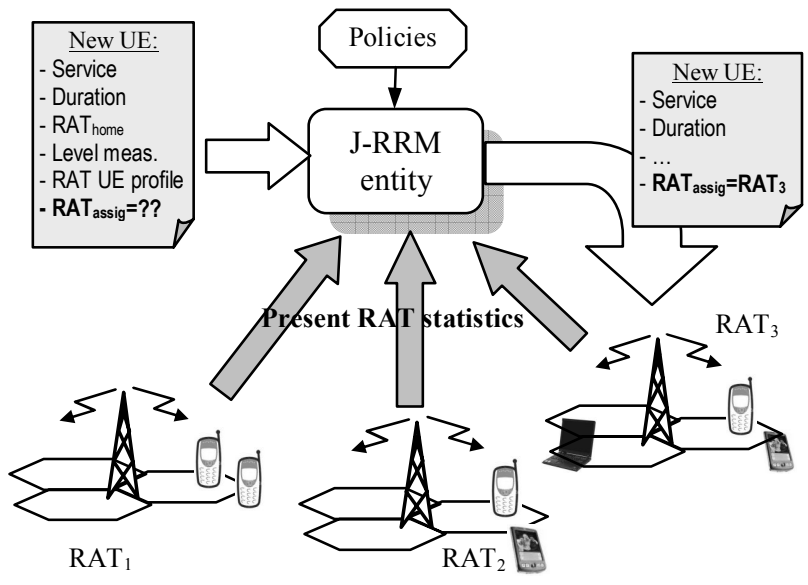

Figure 1. J-RRM decision process
This policy can be seen as a non-joint scheme and it is the current AC algorithm for individual RATs, since there is no cooperation between technologies or resource sharing. In order to compare findings of the proposed AC algorithms, this will be considered as the 'reference' scheme.

The second algorithm $\left(\mathrm{AC}_{\text {type }}=2\right)$ allows for additional attempts after the initial $\mathrm{RAT}_{\text {home }}$ trial. Consequently, redirections are enabled, as reflected in Fig 2. Alternatively, the two remaining $\mathrm{AC}$ methods do not deal with any preferred RAT, but aim to balance the load among RATs. Therefore, UE redirections will be carried out even when the $\mathrm{RAT}_{\text {home }}$ is not completely full. These strategies are adequate to avoid a situation where connections might be blocked due to congestion in high coverage RATs, while low coverage technologies are underutilized, but out of UE's coverage.

As shown in Fig. 3, $\mathrm{AC}_{\text {type }}=3$ assigns the user to the available RAT that is less loaded, while $\mathrm{AC}_{\text {type }}=4$ assigns it to the RAT with the smallest coverage area, regardless the load. Single RAT hierarchical networks, such as GSM, use the same principle to push most connections to cells of lower layers, which provide a discontinuous coverage in the form of 'hot-spots'. Thus, cells of higher layers, which provide continuous coverage, are used to capture the traffic overflow in the lower layers [8]. It is worth noting that these two last algorithms do not make use of the $\mathrm{RAT}_{\text {home }}$ concept, so a significant amount of assignments to $\mathrm{RAT}_{\mathrm{i}} \neq \mathrm{RAT}_{\text {home }}$ will be made. As a consequence, for $\mathrm{AC}_{\text {type }}=3$ and 4 , inter-RAT signalling flows will be quite intense.

\section{SimUlation MODEL}

A simulation environment has been developed to evaluate the above-described AC algorithms. This environment provides a flexible evaluation tool to implement multiple scenarios and compare results. The tool has been developed with the extended MATLAB package.

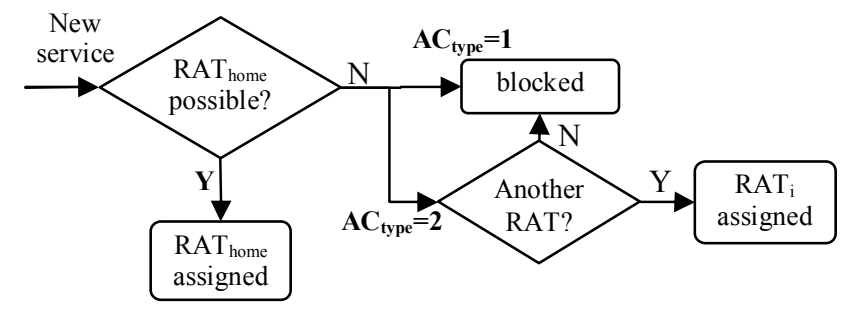

Figure 2. $\mathrm{RAT}_{\text {home }}$ based AC algorithms

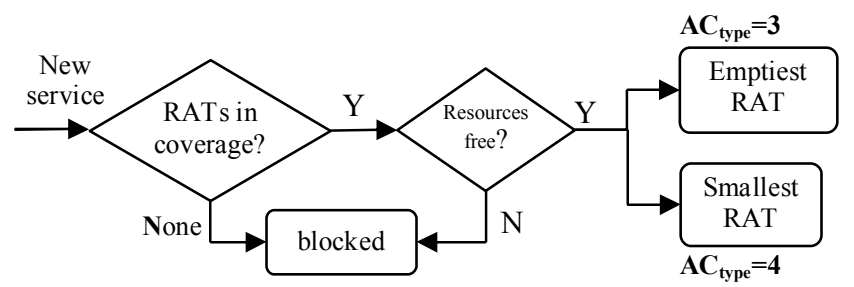

Figure 3. Other admission control algorithms 
To implement the simulator, a time-based approach has been selected, since not only the overall tele-traffic performance, but also the transient response, is relevant. At every iteration, the creation of new connections is evaluated and these connections are addressed to different RATs depending on the AC algorithm. Finally, several statistics are collected to compare distinct network configurations.

1) Scenario: Three technologies have been included in the simulation platform: UMTS-FDD, GSM/GPRS and WLAN. The simulation scenario is represented in Fig. 4. It consists of three different RATs, which provide coverage in a layered structure of three concentric cells [5]. This scenario is the common situation where cells of different RATs share the same site (i.e. are co-sited). The propagation model has been taken from [9] and coverage areas are indicated with a probability index that denotes the proportion of the scenario which is covered by that RAT, $\mathrm{P}_{\mathrm{c}, \mathrm{i}}$. Table I describes a first radio technology covering the whole scenario, i.e. $P_{c, i}=1$, while the others only a portion.

2) Services and user profiles: Table I summarises the main parameters of the simulated services. Different services arrive according to a Poisson traffic model with different rates and their durations follow a negative exponential distribution as Table I shows. Since not every RAT can manage all the services, RATservice matrix indicates, for each service (row), which RAT can be assigned ('1') or not ('0').

The UE multi-RAT capabilities are simulated providing four UE multiRAT profiles with an associated probability, multiRAT $T_{\text {profiles }}$ prob., and a $\mathrm{RAT}_{\text {home }}$ (the first RAT attempt for $\mathrm{AC}_{\text {type }}=1$ and 2), Table I. The users are uniformly distributed over the scenario, and follow a rectilinear trajectory with $90^{\circ}$ random turns. Intersystem-HOs are also implemented.

3) RRM models: A resource management model must be defined for each RAT in order to get reliable results.In UMTS-FDD, a throughput-based admission control scheme has been adopted [4][10]. Each new admitted connection adds its requested bit rate to those of already running connections. If a global bit rate threshold is surpassed, no more connections are accepted.

GSM/GPRS network dedicates its timeslots (TSL) to circuit switched or data switched (DS) connections. TSL distribution is not fixed along the time. It can change depending on both types of traffics necessities. In any case, a maximum number of TSL for DS connections is defined, referred to as GPRS territory, as well as a minimum value, referred to as GPRS permanent.

The coding scheme (CS) adopted for GPRS connections will provide the data rate capacity per TSL [11]. In this work, CS-2 has been assumed, since it is the highest coding scheme currently deployed in live networks. Table II shows the final setting values.

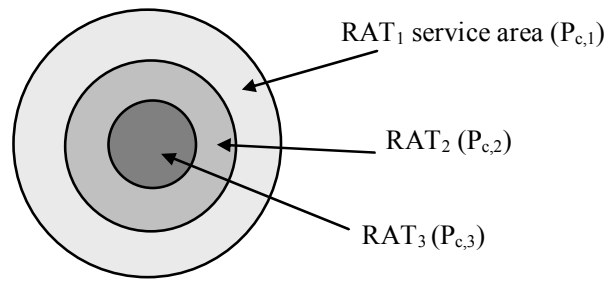

Figure 4. Concentric cells coverage structure

TABLE I. USER, SERVICES AND MOBILITY PROFILE PARAMETER SETTINGS.

\begin{tabular}{|c|c|}
\hline RATs simulated & 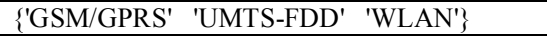 \\
\hline Services & $\begin{array}{l}\{\text { 'Call' 'MMS' 'VideoCall' 'StreamAud' } \\
\text { 'StreamVid' 'Internet' }\}\end{array}$ \\
\hline RATservice & 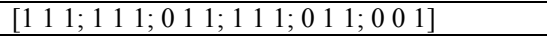 \\
\hline$\lambda_{\text {service }}(\text { user } \cdot \text { sec })^{-1}$ & $1 /\left[\begin{array}{lllllll}1800 & 5000 & 10000 & 10000 & 10000 & 5000]\end{array}\right.$ \\
\hline $1 / \mu_{\text {service }}$ (sec) & {$\left[\begin{array}{lllllllll}10 & 0 & 10 & 100 & 300 & 200 & 600\end{array}\right]$} \\
\hline Data rate $\left(\right.$ kbps $\left._{\text {service }}\right)$ & {$\left[\begin{array}{llllll}12.2 & 4 & 128 & 32 & 128 & 32\end{array}\right]$} \\
\hline $\boldsymbol{P}_{c, i}$ & {$\left[\begin{array}{llll}1 & 0.6 & 0.4\end{array}\right]$} \\
\hline UE multiRAT profiles & 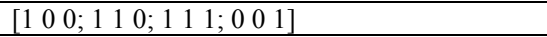 \\
\hline multiRAT $T_{\text {profiles }}$ prob. & {$\left[\begin{array}{llll}0.6 & 0.2 & 0.05 & 0.15\end{array}\right]$} \\
\hline$R A T_{\text {home }}$ & [GSM/GPRS GSM/GPRS UMTS-FDD WLAN] \\
\hline
\end{tabular}

Regarding WLAN technology, an $802.11 \mathrm{~b}$ cell is simulated with Distributed Coordination Function (DCF) mechanism. Its radio resources are shared in a collision avoidance approach (CSMA protocol). This mechanism causes that global throughput to be shared between connections will decrease as more users access to the network [12]. Therefore, maximum and saturation global throughputs are configured as the limits for both low and high traffic situations, as presented in Table II.

\section{RESULTS}

To control the traffic demand, the number of users in the scenario is varied. To asses the value of the different $\mathrm{AC}$ methods, different statistics are used. Concretely, the Call Blocking Rate (CBR) and its complementary, the Call Accepted Rate (CAR), will be used as the main performance indicators to compare different AC algorithms. The CBR stands for the ratio of failed attempts, as in (1).

$$
C B R=1-\text { CAR }=\frac{\text { Blocked Connections }}{\text { Total Attempts }}
$$

TABLE II. GSM, UMTS AND WLAN CARRIER SETTINGS

\begin{tabular}{|c|l|c|}
\hline Technol. & \multicolumn{1}{|c|}{ Parameter } & Value \\
\hline UMTS & Throughput budget & $700 \mathrm{kbps}$ \\
\hline \multirow{2}{*}{ WLAN } & Max Global Throughput & $6.2 \mathrm{Mbps}$ \\
\cline { 2 - 3 } & Saturation Global Throug. & $1 \mathrm{Mbps}$ \\
\hline \multirow{3}{*}{ GSM/GPRS } & Total TSLs & 40 \\
\cline { 2 - 3 } & GPRS permanent/territory & $1-6 \mathrm{TSL}$ \\
\cline { 2 - 3 } & TSL capacity (CS-2) & $12 \mathrm{kbps}$ \\
\hline
\end{tabular}


The ideal Joint AC algorithm would get that no connection is blocked if radio resources are available in any other RAT. Thus, CAR is expected to be improved with better management of shared resources. It will be also interesting to quantify how many connections are accepted in a $\mathrm{RAT}_{\mathrm{i}} \neq \mathrm{RAT}_{\text {home. }}$ This is defined as the Call Redirected Rate (CRR), which indicates the inter-RAT traffic intensity and the amount of signalling exchange between RATs.

To estimate the gain of J-RRM techniques, the previous performance indicators of any AC algorithm must be compared against the non-joint (or reference) scheme. JRRM capacity gain is then calculated as in (2), where subscripts ' $r e f$ ' refers to that of the reference scheme.

$$
\text { Gain }_{\text {JRRM }}=\frac{C A R_{\text {JRRM }}-C A R_{\text {ref }}}{C A R_{\text {ref }}}=\frac{C B R_{\text {ref }}-C B R_{\text {IRRM }}}{1-C B R_{\text {ref }}}
$$

Fig. 5 shows CBR and RAT occupancy with different number of users in the scenario for the first $\mathrm{AC}$ algorithm, i.e. when a connection only tries to be accepted in its $\mathrm{RAT}_{\text {home. }}$ It is worth noting that, in this case, a call is blocked when no radio resources are available, either because of congestion or a lack of coverage. Thus, a clear differentiation between these two components of CBR is established. CBR figures show these two kinds of blockings. When UE is out of $\mathrm{RAT}_{\text {home }}$ coverage, the connection is considered as a 'no coverage' blocking, depicted by the white area in Fig. 5a. As GSM has global coverage, only services with UMTS or WLAN as RAT $_{\text {home }}$ are blocked in this sense. The black area shows the share of connections rejected due to lack of available radio resources. For RATs with low $\mathrm{P}_{\mathrm{c}, \mathrm{i}}$ values (e.g. WLAN), the contribution to the white area is higher, since more UEs are out of coverage (not shown in the graph). As consequence, the contribution of these RATs to the black area decreases, since most connections are generated out of $\mathrm{RAT}_{\text {home }}$ service area.

RAT occupancies in Fig. 5 b clearly indicate that there is free capacity to share between RATs, even for the maximum number of users. The other AC algorithms aim to re-direct those connections that would be otherwise blocked in their $\mathrm{RAT}_{\text {home. }}$ Thus, first failed attempts are not counted as blocked connections, but they are redirected towards other RATs so CBR decreases. Fig. 6 reflects the Gain and CRRs for $\mathrm{AC}_{\text {type }}=2-4$.

Under low traffic conditions, all AC algorithms show similar gain. Real resource sharing is not effective since most of technologies are almost empty. With increasing traffic, third and fourth algorithms show better properties than second. As explained in section II, $\mathrm{AC}_{\text {type }}=3$ and $\mathrm{AC}_{\text {type }}=4$ algorithms are thought to work better for heavy load traffic conditions, at the expense of a larger amount of signaling due to re-directed connections.

$\mathrm{AC}_{\text {type }}=2$ starts to redirect connections only when $\mathrm{RAT}_{\text {home }}$ is full, while the others make redirections before that point. Thus, a load-balancing effect is achieved the latter methods, which overcomes the limitations of methods that only perform congestion control. As observed in Fig. 6, the higher gain of these methods indicates that more connections are carried. Obviously, the gain increases with offered traffic, since the blocking phenomena are more evident.
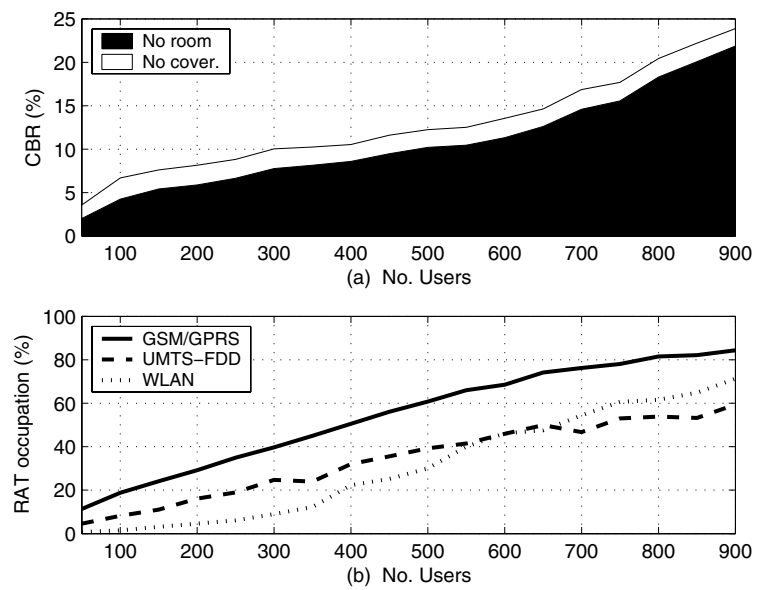

Figure 5. Statistics for a non-joint scheme $\left(\mathrm{AC}_{\mathrm{type}}=1\right)$
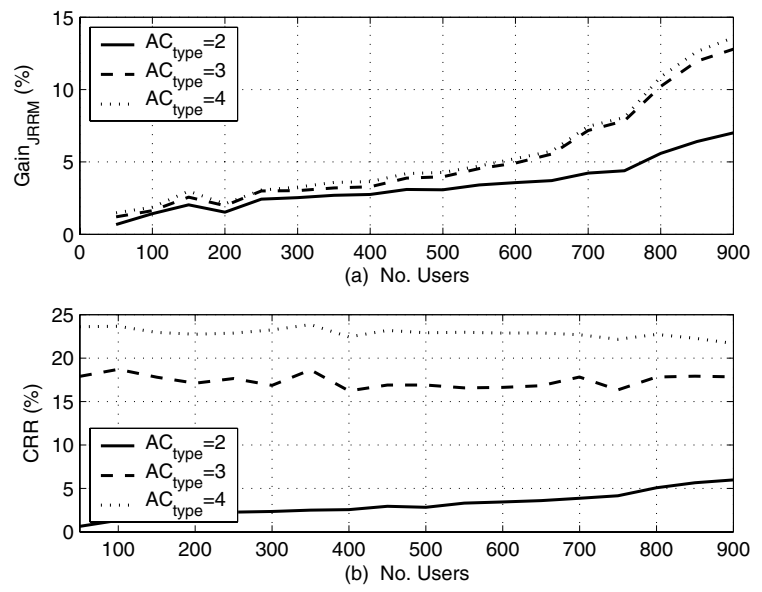

Figure 6. Gain and redirected connections with several AC algorithms

In order to find a significant comparison between $\mathrm{AC}$ algorithms, a $2 \%$ blocking GoS value will be fixed and the number of accepted users in that point will be compared for four different coverage scenarios. Table III presents the different values of coverage probability, $\mathrm{P}_{\mathrm{c}, \mathrm{i}}$, for the different RATs on each case. The coverage in the lower layers (i.e. UMTS and WLAN) range from a small hot-spot (i.e. $\mathrm{P}_{\mathrm{c}, \mathrm{i}}=0.1$ ) to continuous coverage (i.e. $\mathrm{P}_{\mathrm{c}, \mathrm{i}}=1$ ). For a fair comparison, user gain is defined relative to the reference scheme.

The results in Fig. 7 proves that, with low $\mathrm{P}_{\mathrm{c}, \mathrm{i}}$, the inner circles in Fig. 4 have a very small area and only a few connections are actually located in a multi-RAT area. In practice, the area where resources are shared is very limited and no such differences between $\mathrm{AC}$ algorithms are appreciated. When medium and high values of $\mathrm{P}_{\mathrm{c}, \mathrm{i}}$ are 
displayed by all layers, inter-RAT flows start to be significant and different user gains can be reached, specially with $\mathrm{AC}_{\text {type }}=3$ and 4 . The third algorithm gets better results forcing to use resources from technologies with the smallest coverage areas. In other case, these resources could not be completely used for lack of connections being accepted in these RATs. It must be noted that CRR are higher with these two algorithms.

For very high or complete coverage areas, trend changes and second and fourth algorithms behave the same. This behaviour was expected, as $\mathrm{AC}_{\text {type }}=4$ do not re-direct users before congestion, since there is no 'smallest RAT' (i.e. all RATs share the same coverage area). As a result, $\mathrm{AC}_{\text {type }}=3$ is the only strategy that achieves the load-balancing effect and it displays the best figures with $60 \%$ additional users (i.e. $50 \%$ more carried traffic than other JSAC.

\section{CONCLUSIONS}

A general J-RRM network scheme and four AC algorithms have been proposed and evaluated in this paper. The comparison of this structure against the reference or non-joint model $\left(\mathrm{AC}_{\text {type }}=1\right)$ brings benefits with regard to capacity gain and blocking probability. Joint AC algorithms allow full exploitation of existing radio resource at the expense of increasing the signaling load due to a significant number of re-directed connections. Different RAT coverage areas have influence in gain figures since they define the area where resource can be shared. Results show that, under the same blocking conditions, more refined AC algorithms can get around 60-80\% more users accepted in a wide coverage area scenario. Likewise, it has been shown that the selection of the best algorithm is influenced by coverage conditions in the scenario. Nonetheless, $\mathrm{AC}$ algorithms that balance the load among RATs (i.e. $\mathrm{AC}_{\text {type }}=3$-4) provide the best performance figures.

\section{ACKNOWLEDGMENT}

S. L. R. thanks the whole Wireless Access Group in University of Limerick, where this work was partially made.

TABLE III. COVERAGE SCENARIOS

\begin{tabular}{|c|c|}
\hline Scenario & Coverage indexes $\left(\mathbf{P}_{\mathbf{c}, \mathbf{i}}\right)$ \\
\hline Low & {$\left[\begin{array}{lll}1 & 0.3 & 0.1\end{array}\right]$} \\
\hline Medium & {$\left[\begin{array}{lll}1 & 0.6 & 0.4\end{array}\right]$} \\
\hline High & {$\left[\begin{array}{lll}1 & 0.9 & 0.6\end{array}\right]$} \\
\hline Very High & {$\left[\begin{array}{lll}1 & 1 & 1\end{array}\right]$} \\
\hline
\end{tabular}

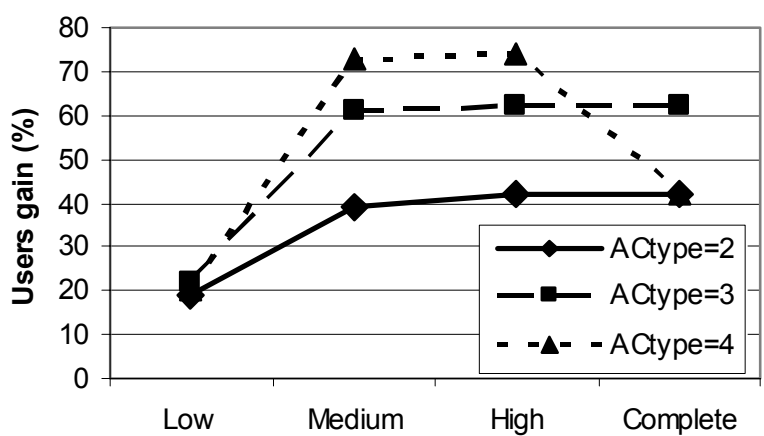

Figure 7. Users Gain for a $2 \% \mathrm{GoS}$ in different coverage scenarios.

\section{REFERENCES}

[1] J. Luo, R. Mukerjee, E. Schulz, M. Dillinger and E. Mohyeldin: "Investigation of Radio Resource Scheduling in WLANs Coupled with 3G Cellular Network". IEEE Communications Magazine; Volume 41, Issue 6, June 2003. Pages:108 - 115.

[2] 3rd Generation Partnership Project. 3GPP TS 22.934 v.6.2.0 "Feasibility study on 3GPP system to Wireless Local Area Network interworking". 2005.

[3] M. O'Callaghan, N.Gawley, M.Barry, S.McGrath: "Admission Control for Heterogeneous Networks". 13th IST Mobile and Wireless Communications Summit, June 27 - 30 2004. Lyon, France.

[4] A. Tölli, P. Hakalin, H. Holma: "Performance Evaluation of Common Radio Resource Management (CRRM)". Proc. of ICC 2002. Volume 5, 28 April-2 May 2002, pages:3429 - 3433.

[5] R. Agustí, O. Salient, J. Perez-Romero, L. Giupponi: “ A fuzzyneural based approach for joint radio resource management in a beyond 3G framework". Procs of QSHINE 2004. 18-20 October 2004. Pages: 216-224.

[6] J. Luo, E. Mohyeldin, N. Motte, M. Dillinger: "Performance Investigations of ARMH ina Reconfigurable Environment"; IST SCOUT 16th Workshop. Paris, September 2003.

[7] N. Motte, R. Rümmler, D. Grandblaise, L. Elicegui, D. Bourse, E. Seidel: "Joint Radio Resource Management and QoS Implications of Software Downloading for SDR Terminals". IST Mobile \& Wireless Telecommunications Summit 2002, June 2002, Thessaloniki, Greece.

[8] X. Lagrange, "Multitier cell design," IEEE Communications Magazine, Volume 35, Issue 8, August 1997, pages:60 - 64.

[9] J. Perez-Romero, O. Salient R. Agustí, M.A. Díaz Guerra: "Radio Resource Management Strategies in UMTS". John Wiley \& Sons, 2005.

[10] H.Holma, A.Toskala, "WCDMA for UMTS," John Wiley \& Sons, $3^{\text {rd }}$ ed., 2005.

[11] T. Halonen, J. Romero, J. Melero, "GSM, GPRS and EDGE Performance - Evolution Towards 3G/UMTS," Johm Wiley and Sons, $2^{\text {nd }}$ ed., 2003.

[12] G. Bianchi, "Performance analysis of the IEEE 802.11 distributed coordination function," IEEE Journal on Selected Areas in Communications, Volume 18, Issue 3, March 2000, page:535 - 547 